\title{
Continual Assessment of Winding Deformation during a Short Circuit Test
}

\author{
S. Santhi $\quad$ Non-member \\ V. Jayashankar ${ }^{* *}$ Non-member
}

A new approach for the continual assessment of winding deformation during short circuit test is proposed. The method is validated through FEM analysis of axially unbalanced axisymmetric configurations. Simulation results are shown to validate the proposed method.

Keywords: winding deformation, concurrent excitation, reactance

\section{Introduction}

The short circuit test on a transformer is a special test as per standards such as IEC $60076 \mathrm{Pt} \mathrm{V,} \mathrm{2000.} \mathrm{The} \mathrm{test} \mathrm{is} \mathrm{an}$ important part in improving design ${ }^{(1)}$. Conventionally, a deviation of winding reactance of more than $2 \%$ (for transformers $<100 \mathrm{MVA}$ ) before and after the test is evidence that the transformer has failed the test. A problem with this assessment is that there is no indication of failure during the test itself. Test authorities measure the reactance before and after the test ${ }^{(2)}$. A method of online assessment of winding deformation was proposed based on the superposition of a high frequency signal concurrently with the power frequency current $^{(3)}$. For some configurations, the winding current resonant frequencies may be too high and we hence propose the injection of a periodic measuring signal (at $800 \mathrm{~Hz}$ ) for continual assessment of reactance.

\section{Device Under Test}

In order to establish the method from first principles we have chosen a configuration that demonstrates winding deformation in a simple but effective manner. The arrangement (often called a jumping ring) is shown in Fig. 1. The problem has attracted considerable attention from a computational viewpoint and is solved using a $3 \mathrm{D}$ transient eddy current formulation ${ }^{(4)}$.

It can be shown that with appropriate excitation the shorted secondary (the ring) experiences vertical force that causes it to lift. In terms of transformer parlance the configuration has unbalanced ampere-turns that exhibits considerable axial force. The primary winding has 850 turns, outer diameter of $9 \mathrm{~cm}$ and an axial length of $10 \mathrm{~cm}$. The core length is $18.5 \mathrm{~cm}$ and the area is $2.8 \mathrm{~cm} \times 2.4 \mathrm{~cm}$. The secondary winding is 10 turns, 12 gauge coil of mass $50 \mathrm{~g}$. The outer diameter is $6.6 \mathrm{~cm}$ and has an axial length of $1 \mathrm{~cm}$.

\section{Computation of Axial Force}

The configuration of Fig. 1 was analyzed using MAGNET, a finite element package that solves Maxwell's equation in differential form. The input excitation was increased until the resulting change in impedance at the end of $0.5 \mathrm{~s}$ was about $2 \%$. The value of this input excitation is $0.4 \mathrm{~A}(\mathrm{rms})$ and is

\footnotetext{
* Research Scholar, Electrical Engg., IIT Madras

Chennai-600036, India

** Dept. of Electrical Engineering, IIT Madras

Chennai 600 036, India
}

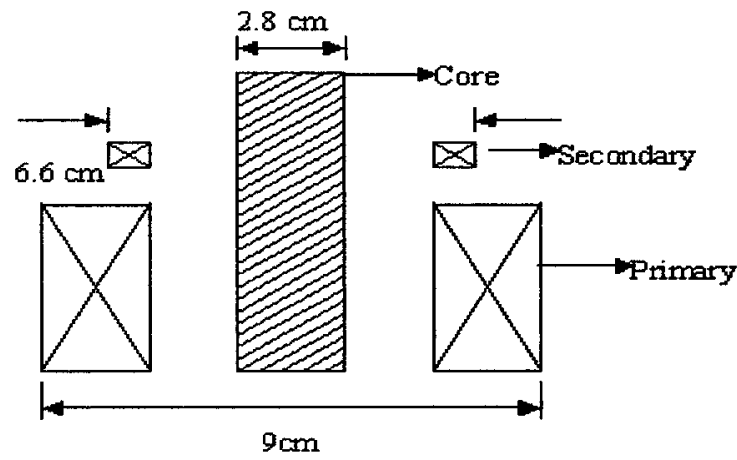

Fig. 1. Schematic diagram of the device under test
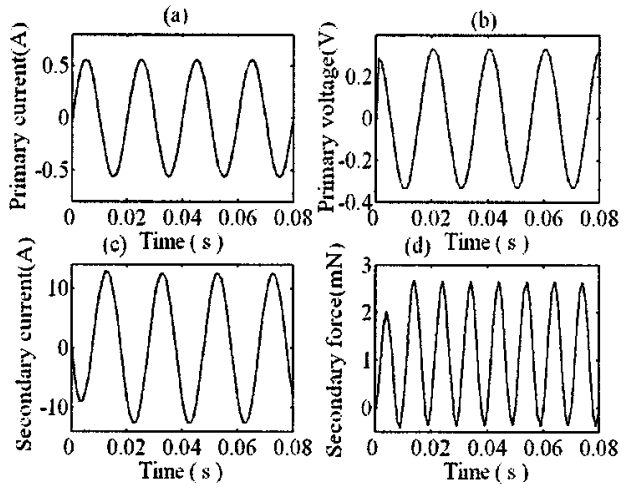

Fig. 2. Primary current, voltage, secondary current, force

from a $50 \mathrm{~Hz}$ current source. The input primary current excitation (a), voltage across the primary (b), secondary current (c), and the force on the secondary ring (d) are plotted in Fig. 2. Only 4 cycles of the input are shown for convenience.

4. Assessment of Winding Reactance

In order that the axial displacement of the secondary ring can be identified online, we propose the measurement of impedance on a cycle-by-cycle basis. Figure 3(a) shows the position of the ring at different time instants. The impedance is computed from the primary voltage and current records. Figure 3(b) shows the cycle-to-cycle \% variation of impedance with time. We are thus able to assess deformation continually and the method can be used with existing measurement techniques during short circuit testing. 


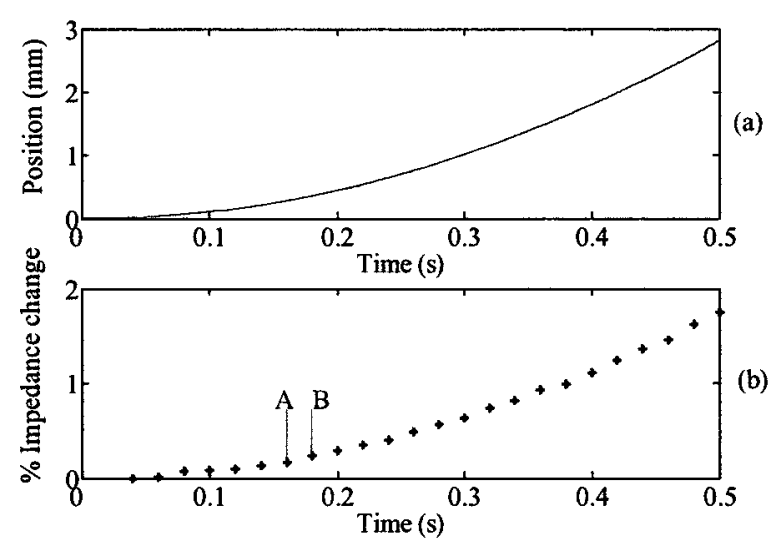

Fig. 3. (a) Position of ring, (b) impedance (computed on a per cycle basis)

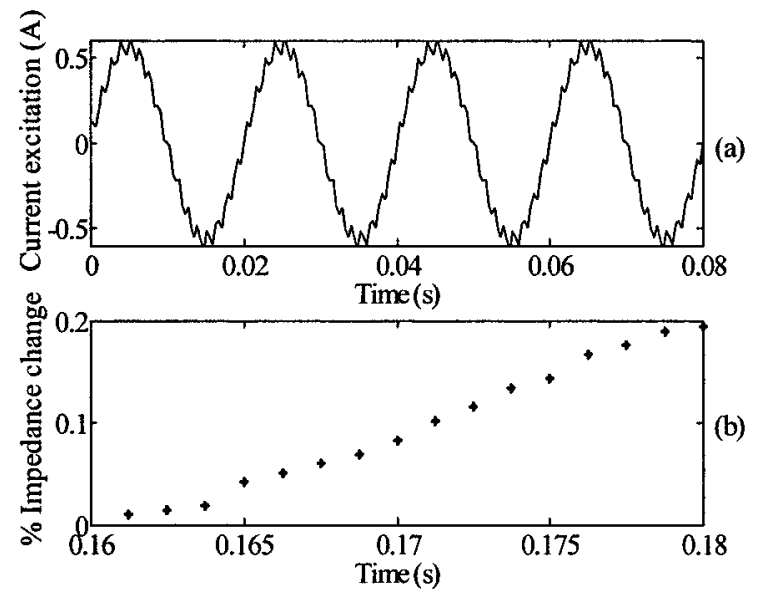

Fig. 4. (a) Primary current excitation $(50 \mathrm{~Hz}$ plus $800 \mathrm{~Hz}$ ), (b) cycle to cycle \% Impedance variation at $800 \mathrm{~Hz}$

\section{Improving Resolution of Reactance Measurement}

In order to improve the resolution of the method, we propose the injection of a periodic sinusoidal signal $(800 \mathrm{~Hz})$ on the $50 \mathrm{~Hz}$ power frequency so that the impedance can be measured in a shorter duration. The amplitude of the additional periodic signal is small and just sufficient for measurement purposes. It will not affect the short circuit test. Accordingly the excitation was changed as

$$
i(t)=0.5656 * \sin (\omega t)+0.05656 * \sin (16 \omega t)
$$

where $\omega$ corresponds to $50 \mathrm{~Hz}$ and is shown in Fig. 4(a) (only 4 cycles shown for convenience). This ensures that in one cycle of a $50 \mathrm{~Hz}$ power frequency 16 measurements are possible and over the duration of $0.5 \mathrm{~s}$ we can thus have 400 values for impedance. Fig. 4(b) shows the changes in impedance at $800 \mathrm{~Hz}$ during a cycle of the $50 \mathrm{~Hz}$ waveform between $0.16 \mathrm{~s}$ and $0.18 \mathrm{~s}$ corresponding to points A and B of Fig. 3(b).

\section{Equal Concentric Windings}

To consider a more realistic case two concentric coils are considered as shown in Fig. 5. The coils have equal axial length of $10 \mathrm{~cm}$, equal turns of 850 . The outer diameter of

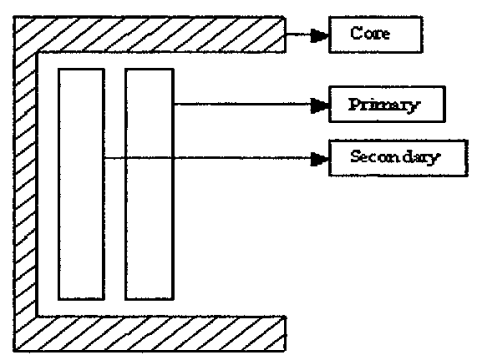

Fig. 5. Schematic diagram of concentric winding

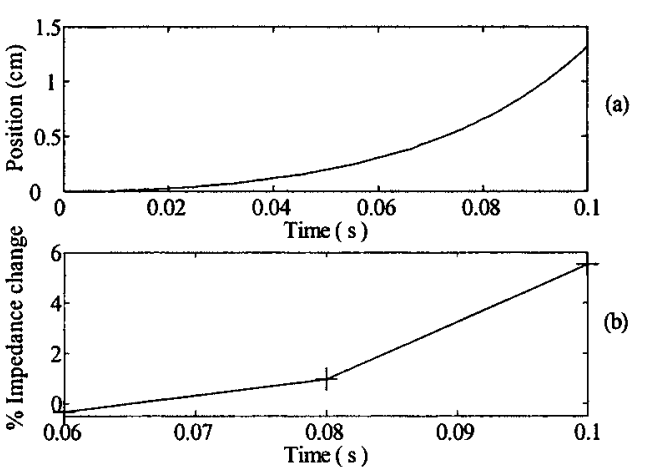

Fig. 6. (a) Position of secondary, (b) impedance (cycle to cycle)

primary is $14 \mathrm{~cm}$ and that of secondary is $8 \mathrm{~cm}$. The core axial length is $24 \mathrm{~cm}$. The secondary is initially displaced axially from the primary by $0.7 \mathrm{~cm}$ in order to create unbalanced ampere-turns. The primary coil excitation is increased as before until the change in impedance is about $2 \%$ within 5 cycles of a $50 \mathrm{~Hz}$ waveform. The $\%$ change in impedance is determined at different time instants. The position of secondary and the $\%$ variation of cycle-to-cycle impedance are shown in Fig. 6(a) and Fig. 6(b) respectively. A continual assessment of impedance with external measurements alone can thus yield information on winding deformation without untanking.

\section{Conclusion}

A new approach for the continual assessment of winding deformation during a short circuit test was proposed. It was validated through FEM analysis of configurations that exhibit unbalanced ampere-turns.

(Manuscript received Feb. 17, 2006)

\section{References}

( 1 ) T. Olsson: "800 kV AC Transformers, Built for reliability", Trafotech 2006, pp.I 1-6, Mumbai, India (2006)

( 2 ) R.P.P. Smeets and L.H.te Paske: "An Update test experiences with shortcircuit withstand capability of large power transformers", Trafotech 2006, pp.III 31-38, Mumbai, India (2006)

( 3 ) S. Santhi, S. Jayalalitha, and V. Jayashankar: "Detection of Winding deformations during short time current tests", IMTC -2005, Ottawa, Ontario, Canada (2005)

( 4 ) J.P. Webb, B. Forghani, and D.A. Lowther: "An Approach to the solution of three dimensional voltage driven and multiply connected eddy current problems”, IEEE Trans. Magnetics, Vol 28, No.2, pp.1193-1196 (1992) 